TZGHICAL INFOHATION ON BUIIDING RAMERIAIS

FOR USE IN THE DESIGI OF LOH-COST HOUSING

TIBM-9

\author{
$* * * * * *$ \\ THE ITATONAI BURTAU OF STAITDARDS \\ TIITED STATES DEPARTIENT OF COMERCE \\ TASHINGROI, D. C.
}

\title{
May $13,1936$.
}

\section{CONCDOTE FLOOR MREATMENTS}

This is a digest of information found in Letter Circular 139, "Reoort of Service Tests on Concrete Floor Treatments", (October 28 , 1920), I issued by the Bureau of Standards.

A comparative study of 17 proprietary and 5 "home" treatments was made, based upon observations of treatnents applied to corridor panels 8 feet square whicin were all subjected to much the sane foot traffic concitions. The first tieatments were applied about 5 months after the corridor floors were completed, at which tirue they had bogun dusting. Other treatments folloved durine the next 6 montins.

While the results wore not quantitative or necessarily conclusivo, they wore gonerally indicative of what might be expected with regard to service and behavior.

The summary following shows that treatments A to inclusive gave generally good results but that further study was needed to determine the proper strensth of the magnesium fluosilicate solution and methods of apolication. Treatment $G$ gave excellent results. "Eomell treatments I and $\mathrm{J}$ proved very successful, were easily applied and inexpensive. Instructions for their preparation and use are given on page 6 .

Iobtainable without charce from the National Bureau of Standards, Mashington, D. C. 



\section{CONDITION AND APPEARAITCE OF TREATED COITCRETY FIOOR PANELS}

\section{AT END OI SERVICE PHEIODS}

\begin{tabular}{|c|c|c|c|c|}
\hline $\begin{array}{l}\text { Treat- } \\
\text { ment }\end{array}$ & $\begin{array}{l}\text { Composition } \\
\text { and } \\
\text { lifethod of Application }\end{array}$ & $\begin{array}{l}: \\
: \text { Service } \\
: \text { Pcriod } \\
:\end{array}$ & $\begin{array}{l}\text { : Conaition } \\
: \text { and } \\
: \text { Appearance }\end{array}$ & 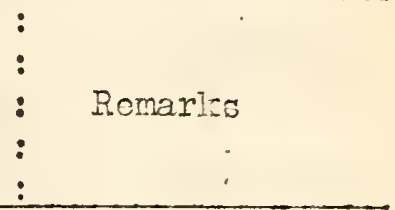 \\
\hline A & 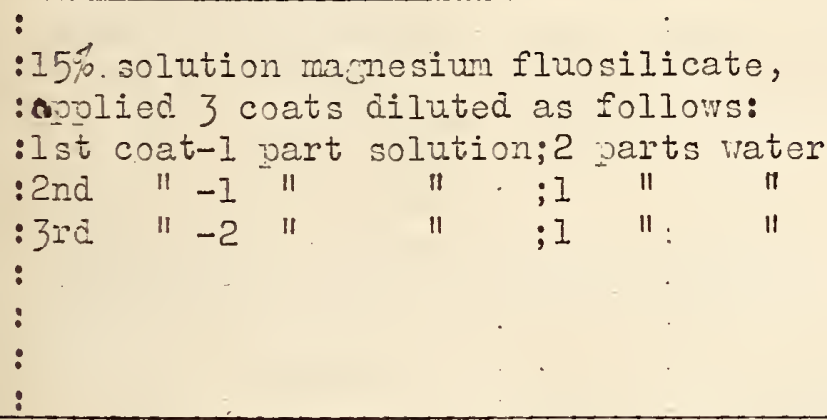 & 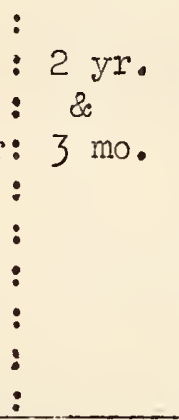 & $\begin{array}{l}\text { : Surface } \\
\text { : quite hard. } \\
\text { : A few small, } \\
\text { : soft, and. } \\
\text { :readily } \\
\text { : scratched } \\
\text { :areas show } \\
\text { : signs of } \\
\text { :wear. }\end{array}$ & $\begin{array}{l}\text { : Appears these } \\
\text { :areas originally } \\
\text { :received improper } \\
\text { : amount of treat- } \\
\text { :ment. } \\
: \\
: \\
:\end{array}$ \\
\hline$B$ & $\begin{array}{l}: \\
: 8.7 \% \text { solution magnesium fluosilicate, } \\
\text { :apolied } 3 \text { coats diluted same as } \\
\text { :Treatment } A, \text { regarciless of voalier } \\
\text { :solution. }\end{array}$ & $\begin{array}{l}: \\
: 1 \text { yi. } \\
: \text { \& } \\
: \quad 9 \text { ino. }\end{array}$ & $\begin{array}{l}\text { :Considerable } \\
\text { :wear. } \\
\text { : Scratched in } \\
\text { :many places. }\end{array}$ & $\begin{array}{l}\text { :Probable that } \\
\text { : solution was too } \\
\text { :veak. }\end{array}$ \\
\hline C & $\begin{array}{l}: 14.5 \% \text { solution magnesium fluosilicate, } \\
\text { :applied copiously in l coat without } \\
\text { :dilution. } \\
: \\
:\end{array}$ & 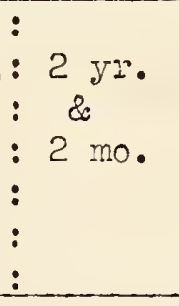 & $\begin{array}{l}\text { :Good conai- } \\
\text { :tion. } \\
\text { :Uniform in } \\
\text { :apoearance } \\
\text { :No sions of } \\
\text { :wear. }\end{array}$ & $\begin{array}{l}\vdots \\
\vdots \\
\vdots \\
\vdots \\
:\end{array}$ \\
\hline$D$ & $\begin{array}{l}: \\
: 11.5 \% \text { solution mamesium fluosilicate, } \\
\text { :aplied } 3 \text { coats diluted same as } \\
: \text { Mreatment A. } \\
:\end{array}$ & $\begin{array}{l}: \\
: 1 \mathrm{gr} . \\
: \quad 8 \mathrm{mo} \\
:\end{array}$ & $\begin{array}{l}\text { :No cêinite } \\
\text { : signs of } \\
\text { : wear. } \\
\text { :Tniform } \\
\text { :appearance. }\end{array}$ & $\begin{array}{l}: \\
: \\
: \\
: \\
:\end{array}$ \\
\hline$\Xi$ & $\begin{array}{l}: \text { :I8\% solution masnesium fluosilicate } \\
\text { :and small amount zinc fluosilicato, } \\
\text { :applied } 3 \text { coats diluted same as } \\
\text { :Treatment A. }\end{array}$ & $\begin{array}{l}: \\
: \\
\vdots \\
\vdots\end{array}$ & $\begin{array}{l}\text { :No apprecia- } \\
\text { :ble wear. } \\
:\end{array}$ & $\begin{array}{l}\text { :Applied to a vor } \\
\text { :poor panel, i.e., } \\
\text { :crumblins bady } \\
\text { :at the surface. }\end{array}$ \\
\hline $\bar{F}$ & $\begin{array}{l}: 7.3 \% \text { solution masnesium fluosilicate, } \\
\text { :cntaining } 2.6 \% \text { magnesium sulphate } \\
\text { :and } 4.5 \% \text { free nydrowluosilicic acid, } \\
\text { :apolied } 3 \text { coats diluted same as } \\
\text { :Treatment A. }\end{array}$ & $\begin{array}{l}: 1 \mathrm{yr} . \\
: \quad \& \mathrm{mo} \\
:\end{array}$ & $\begin{array}{l}\text { :Consiclerable } \\
\text { :wear. } \\
\text { :Easily } \\
\text { : scratched. } \\
\text { : }\end{array}$ & $\begin{array}{l}: \\
\vdots \\
\vdots \\
:\end{array}$ \\
\hline
\end{tabular}




\section{AT END OI SERVICE PERIODS (Cont.-2)}

\begin{tabular}{|c|c|}
\hline $\begin{array}{l}\text { Treat- } \\
\text { ment }\end{array}$ & $\begin{array}{cc}: & \text { Composition } \\
: & \text { and } \\
: & \text { Methoc of Application }\end{array}$ \\
\hline$G$ & $\begin{array}{l}: \\
: 16 \% \text { solution zinc sulphate with about } \\
: 4.5 \% \text { free sulphuic acid, apliea. } 2 \\
: \text { oats without dilution. } \\
: \\
:\end{array}$ \\
\hline $\mathrm{H}$ & $\begin{array}{l}: 20 \% \text { solution sodiun silicate with } \\
: \text { small acdition of an oronic acia, } \\
\text { applied } 2 \text { coats without dilution, } \\
: 24 \text { hours apart. } \\
:\end{array}$ \\
\hline$I *$ & $\begin{array}{l}: 8 \% \text { solution commercial sodium } \\
: \text { silicate, apolied } 3 \text { coats. } \\
: \\
: \\
:\end{array}$ \\
\hline
\end{tabular}

$J *: 15 \%$ solution aluninum sulphate,

\begin{tabular}{|c|c|c|}
\hline : & : & : \\
\hline : Service & Condition & : \\
\hline :Period & and & Remarks \\
\hline : & : Appearance & : \\
\hline & : & ; \\
\hline & & : \\
\hline
\end{tabular}

: \& and uiform : 4 hrs. Surface

: 3 mo. : surface. : then scrubbed

: :Darler than :with hot water

: :orioinal :and mopoed dry

: :concrete. :before 2nc coat

$: \quad:$

: $:$ applied.

: 2 yr. :Ito signs of :Slab covered wi : \& :wear. :plank until àry

: 2 mo. :Surface hard:brichter and

: and uniform. snore uniform

: : :appearance than

$\therefore \quad$ :____original.

: $2 \mathrm{Jr}$ : :ITo signs of : Each coat was

: \& :wear. Very :preceded by

: 2 no. :hard surface: thorough scrub-

: :Uniform :bins with water

: :appearance, :

: :lishter than:

: : original.

: : :

:applied 3 coats äiluted as follows: \& :as other :ally with white

:1st coat-1 part solution; 2 parts water: 6 mo. :panels but :wash brush;

:2nd " -1 " " 1 " " $"$ "

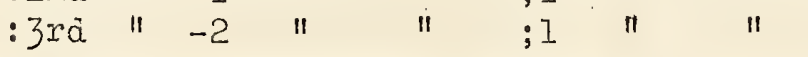

\section{:}

$:$

:

:

$\mathrm{K}$ : Gray paint with pi fment of basic lead : sulphate, siliceous matter and carbon :in tung oil resin varnish (mineral : spirits thinner), applied 2 coats, $: 24$ hours apart.

\section{: factory.} :hrs. Very ecom

$:$

$\therefore$ :nomical horne : treatinent.

: 2 yr. : Shows effect: Surface thorougl

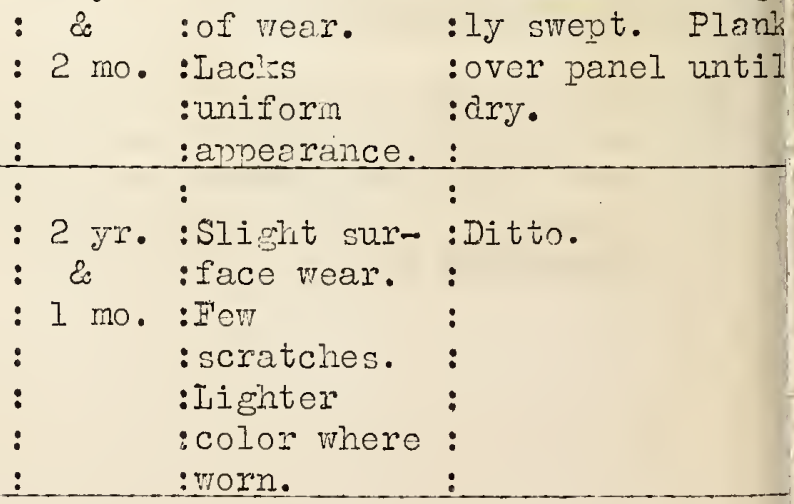




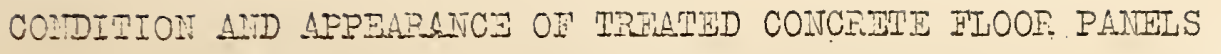 AT IDO OF SERVICI PERODS (cont.-3)}

\begin{tabular}{|c|c|c|c|c|}
\hline $\begin{array}{l}\text { Ireat- } \\
\text { mont }\end{array}$ & $\begin{array}{c}\text { Composition } \\
\text { aird. } \\
\text { liethod of Apulication }\end{array}$ & $\begin{array}{l}: \text { Service } \\
: \text { Period } \\
:\end{array}$ & $\begin{array}{l}: \text { Conaitirn } \\
: \quad \text { and } \\
:\end{array}$ & $\begin{array}{l}: \\
\vdots \\
:\end{array}$ \\
\hline $\mathbb{U}$ & $\begin{array}{l}\text { :Same as Tiveatment I. } \\
: \\
:\end{array}$ & $\begin{array}{l}: 2 \mathrm{yr} . \\
: \& \mathrm{mo} . \\
:\end{array}$ & $\begin{array}{l}: \text { ino appre } \\
\text { :ciable signs } \\
\text { :of vear. } \\
:\end{array}$ & $\begin{array}{l}\text { :Surface } \\
\text { : thoroughty } \\
\text { : swept. Plank } \\
\text { :over panel } \\
\text { :until dxy. }\end{array}$ \\
\hline it & $\begin{array}{l}\text { :Thin bodied minenal spirits varnish, } \\
: \text { apilea } 2 \text { ccats, } 24 \text { hours apart. } \\
: \\
:\end{array}$ & $\begin{array}{l}: 2 \mathrm{yr} \\
: 2 \mathrm{mo} \\
: \\
:\end{array}$ & $\begin{array}{l}\text { : Signs of } \\
\text { : vear denoteo } \\
\text { :by light } \\
\text { :appearance. } \\
:\end{array}$ & $\begin{array}{l}\text { :Ditto. Panel } \\
\text { :originally weal } \\
\text { :and crumbling } \\
\text { :badly, hence } \\
\text { :test quite } \\
\text { : severe. }\end{array}$ \\
\hline 0 & 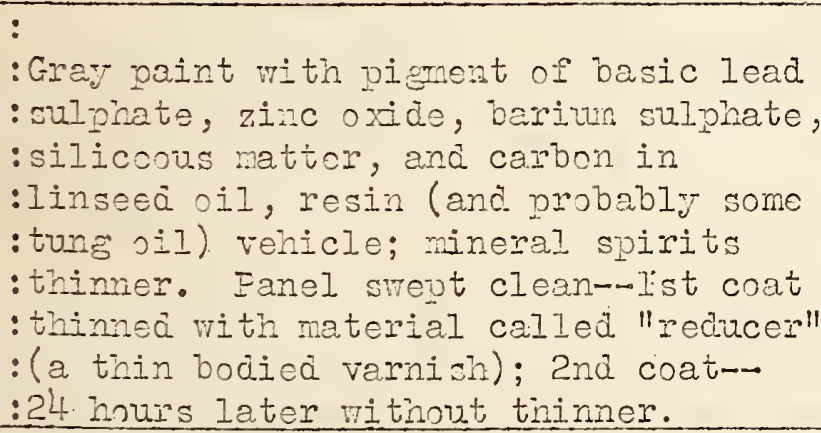 & $\begin{array}{l}: 1 \mathrm{yr} \\
: 5 \mathrm{mo} \\
: \\
: \\
: \\
:\end{array}$ & $\begin{array}{l}\text { :ito signs of } \\
\text { :wear excopt } \\
\text { :few } \\
\text { :scratches } \\
\text { : on wax-like } \\
\text { : surface. } \\
: \\
:\end{array}$ & $\begin{array}{l}\text { : Not especially } \\
\text { :rosistant to } \\
\text { : scratching but } \\
\text { :reasonably } \\
\text { : durable urider } \\
\text { : foot traffic. } \\
: \\
:\end{array}$ \\
\hline$I$ & $\begin{array}{l}\text { :Very thicl paint consisting of pienent } \\
\text { :f zinc oxidc, lithophono and bone } \\
\text { :olack in varnish vohicle containing } \\
\text { :resim, applied in } 1 \text { coat, sufficient } \\
\text { :for purpose. }\end{array}$ & $\begin{array}{l}1 \mathrm{Jr} \\
: \\
: \\
: \\
:\end{array}$ & $\begin{array}{l}\text { :Thicl film } \\
\text { :marred by } \\
\text { : small spots } \\
\text { :blistered } \\
\text { :and worn } \\
\text { :off. }\end{array}$ & $\begin{array}{l}\text { : Pleasing to } \\
\text { :walk on but has } \\
\text { :not pioven } \\
\text { : durable. Dircc- } \\
\text { :tions callea } \\
\text { :fnr two coats. }\end{array}$ \\
\hline$Q$ & $\begin{array}{l}\text { Solution of heavi hydro-carbon wax in } \\
\text { :light hydro-carbon oil, applied } 2 \\
\text { :coats, } 24 \text { hours apart. } \\
: \\
:\end{array}$ & 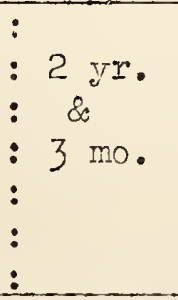 & $\begin{array}{l}: \\
\text { :Consiocrabl } \\
\text { :wear. } \\
\vdots \\
\vdots \\
\vdots\end{array}$ & $\begin{array}{l}\text { :This treatment } \\
\text { :only to hnla } \\
\text { : dust domn. Nis } \\
\text { :claims macic as } \\
\text { :to hardcning } \\
\text { : surface. }\end{array}$ \\
\hline R & $\begin{array}{l}: \text { Mixture of waxes applied in molten } \\
\text { :conditinn. Surface heated before } \\
\text { :and after aoplication. } \\
:\end{array}$ & $\begin{array}{l}: 2 \mathrm{Jr} \\
: 8 \mathrm{mo} \\
:\end{array}$ & $\begin{array}{l}\text { : Shows con- } \\
\text { : siderable } \\
\text { : wear; worn } \\
\text { : through un- }\end{array}$ & $\begin{array}{l}\text { :Treatment aw- } \\
\text { :plifd to } 1 \\
\text { :panel and } 1 \\
\text { :office room. }\end{array}$ \\
\hline
\end{tabular}




\section{CONDITION AND APDEARANCE OF TREATED CONCREIIT FLOOR PANETS}

\section{AT BND OF SERVICE PFITIODS (Cont.-4)}

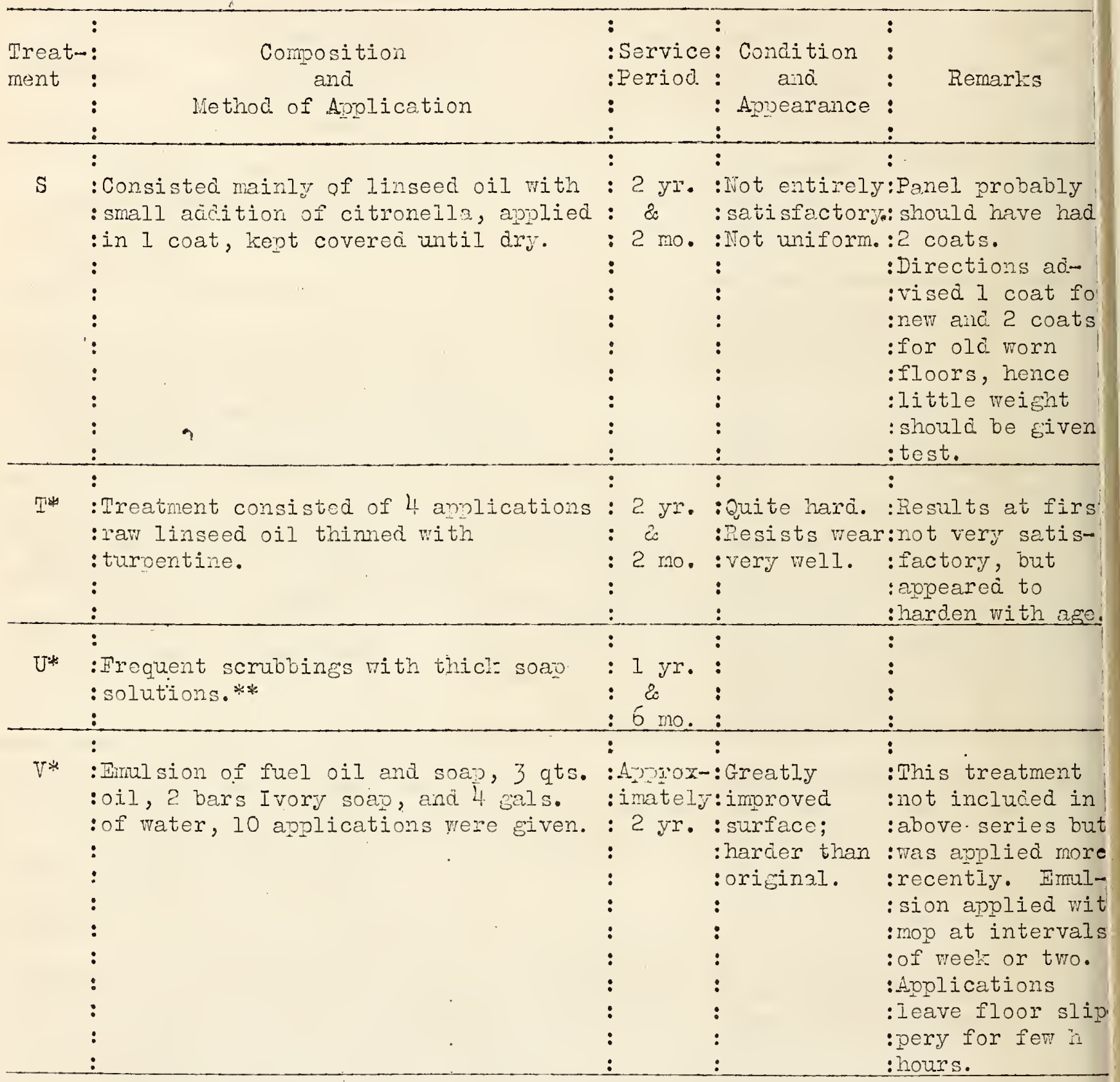

\section{*Incicates horne treatrents.}

**Treatment U: Concrete floors unser actual use sometineș take on a polished or war-like appearance. To determine if precinitation of soan in the concrete caused this, sections of floor were frequently scrubed with a thick soap solution. The polished condition did not cccur in this case, probably due to floor being very porous, hence, the solid matter from treatment was not retained in the concrete. 
Instructions for mating two of the lome treatments:

(I) Sodium Silicate Treatment: Dilute each gallon of comnercial

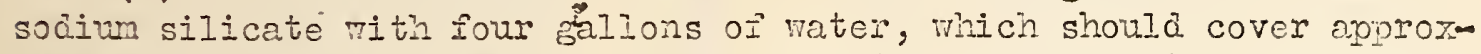
irately 1000 square feet, one coat, ciependine on porosity of floor to be treated. The solution should not be mixed until ready for irmediate use and then applied with mov or hair broou, continuously brushing surface for several ininutes to obtain an even penetration.

Before aploring treatment, all brease spots, plaster, etc., shoula be tinoroughy removed from tine surface to be treatec, scrubbed with clcar rater and then cirłea several days.

Trentj-four hours should be allowed jetween applications, scmionbing with clear rater between each treatment. Three applications should, in most cases, prove sufficient, but if saturation point does not seem to have been completely reached, a fourth coat should be aoplied.

(J) Aluminum Sulphate Treatment: Solution should be macie in mooden barrel or stonetara vessel. Estinate one gallon of solution for each 100 square feet of area. To melse solution, dissolve $21 / 2$ pounds of powdered aluminum sulphate per gallon of vater, aciculating the rater by aiding $2 \mathrm{cc}$. (about 40 drops) of comiercial sulphuric acid. The solution shoulc be stirred occasionaliy for a few dajs until completely dissolved.

Thoroughly ury clean and scrub floor as cirected in ireatment I. After the surface has dried and at 24 hour intervals, aoply 3 treatw ments, mixing for the lst coat, 1 part solution to 2 parts water; 2nd coat, I part solution to I part vater; 3rd coat, 2 parts solution to 1 part water. Apply with mop or hair broom, brushing for several minutes to secure uniform penctration. After 3rd coat has dried, scrub with hot water. 
